\title{
A Home-made Balanced Mode Differential Detector
}

\author{
S. Ghezali and M. Kasevich* \\ Physics Department. Faculty of sciences. University of Blida. Blida. Algeria. \\ *:Sloane Physics Laboratory, Physics Department. Yale University. New Haven. CT. USA.
}

\begin{abstract}
A low noise differential detector has been constructed in order to reduce the laser shot noise. A factor of $30 \mathrm{~dB}$ of the reduction of the rejection noise has been obtained by simulating the amplitude modulation of two laser probes with a home-made electro-optical modulator (EOM). Cold atom devices have a detection zone which may be non-separated from the trapping/cooling zone. The aim of this short letter is to demonstrate the power of a homodyne balanced mode differential detection in a cold atom device experiment and to extrapolate it in a modulation transfer spectroscopy technique. A differential detector has been built with two identical Silicone photodiodes mounted as shown in figure 1 [1]. The photocurrent derived from both photodiodes is controled by the interfet transistor and amplified with a one stage low noise amplifier.
\end{abstract}

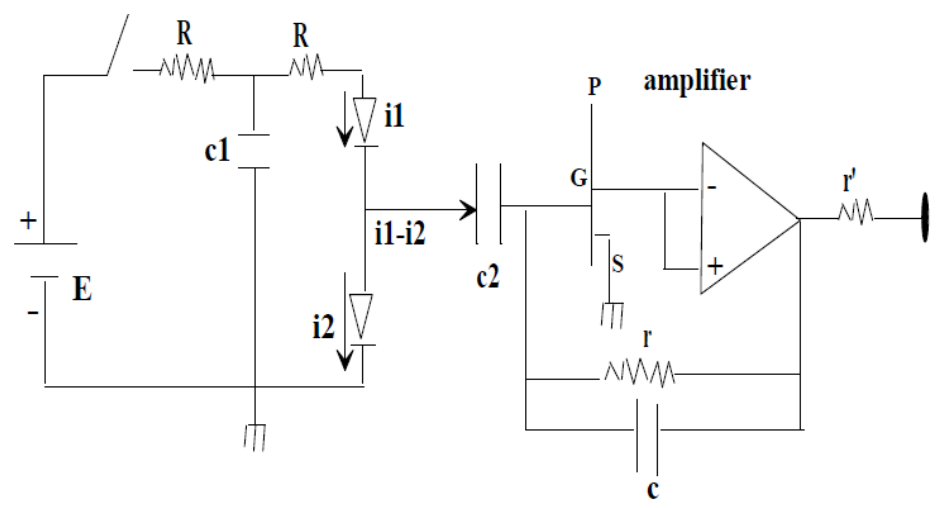

Figure1: A balanced mode differential detector

We can envisage to work with a home made electro-optical modulator (EOM) constituted by a conductor wire rolled around a cylindrical ceramic which plays the role of a capacitor fixed between two metallic pieces. We choose a resonance frequency $(\sim \mathrm{MHz})$ by adjusting the mechanical pressure (for example with many screws) applied on the upper mechanical piece.

The microwave energy is introduced inside the box via a SMA connector [2]. See figure 2.

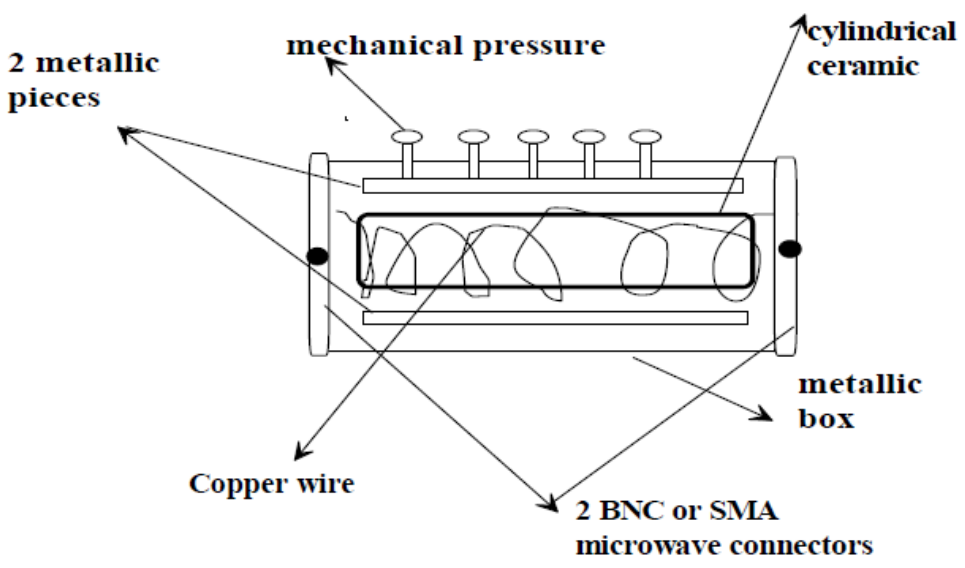

Figure 2: A Home Made Electro-Optical Modulator.

Otherwise, a very good quality resonant EOM can be available in the market. The wide band EOM are in fact less performant. 


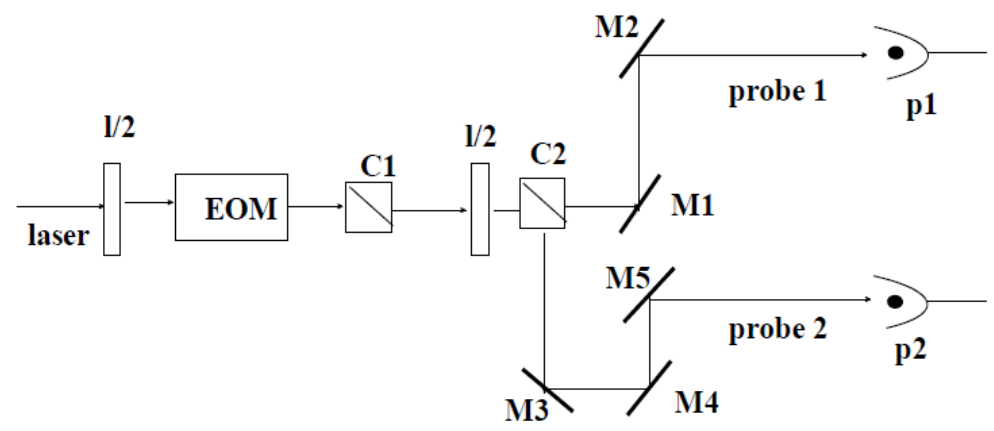

Figure 3: Amplitude modulation of two probe lasers.

The use of the EOM is simple. It is intended to undergo an amplitude modulation of two probe lasers. A probe laser is modulated in amplitude via the EOM (to few $\mathrm{MHz}$ ) and is divided into two probes in order to undergo a differential detection.

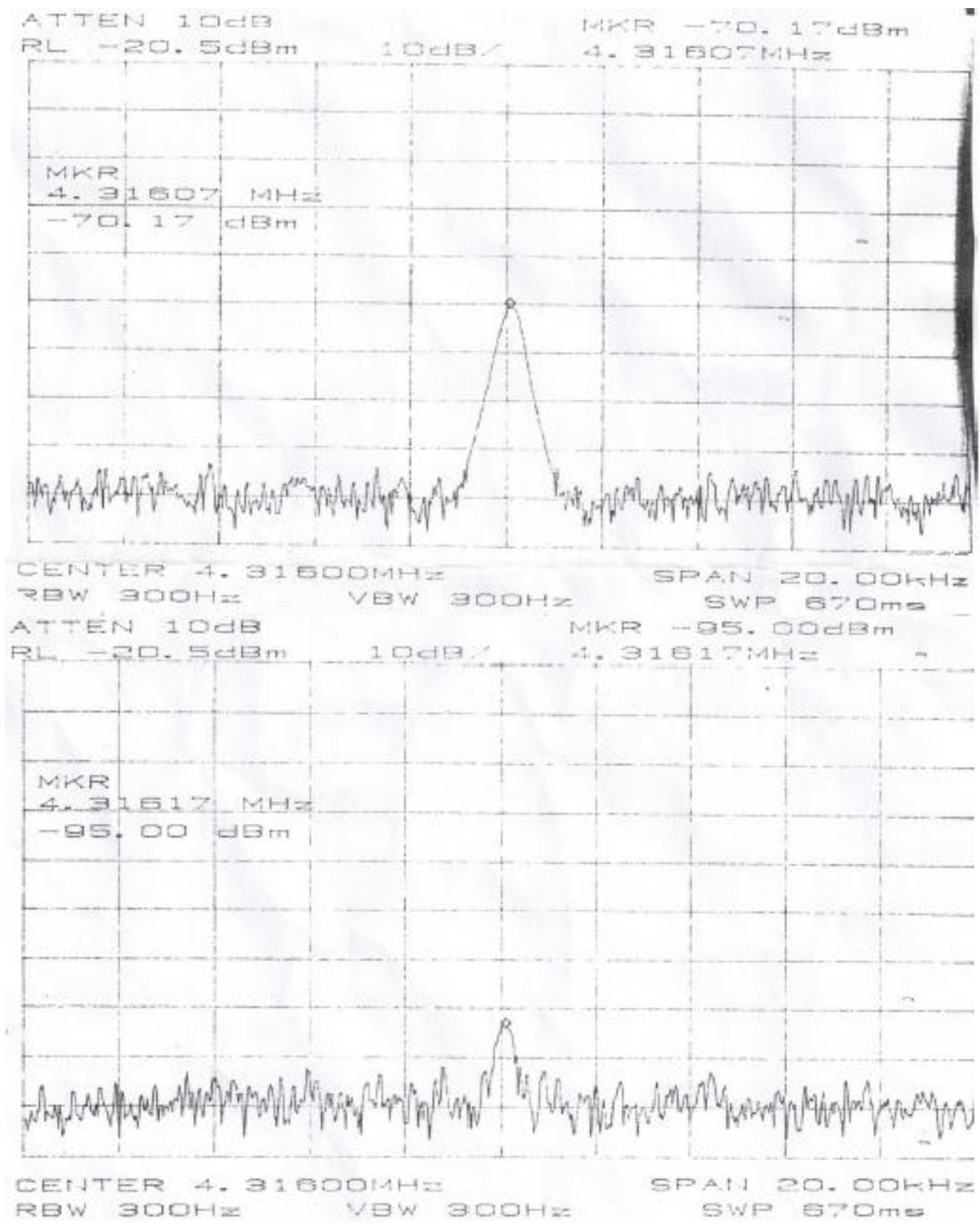

Figure 4: Reduced signal of a factor of $30 \mathrm{~dB}$. 
This will reduce the detection noise, essentially the photons' noise taken back to the intrinsic noise of each photodiode $[3,1]$ (see figure 4 ). We note that the reduction factor of the photons' noise can be affected by the thermal drift of the modulator.

This work has been undergone at the Sloane Physics Laboratory at Yale university in 1999 and has never been published.

\section{References}

[1] P. C. Hobbs, Reaching the shot noise limit, Optics \& Photonics News, 17-23, Avril 1991.

[2] S. Ghezali, Private communication, 2000.

[3] J. M. McGuirk, G. T. Foster, J. B. Fixler and M. A. Kasevich, Opt. Lett., Vol. 26, Issue 6, Mars 2001. 